\title{
INTERCALIBRATION OF AIRGLOW OBSERVATORIES WITH THE ATMOSPHERE EXPLORER SATELLITE
}

\author{
MARSHA R. TORR*, P. B. HAYS and B. C. KENNEDY \\ Space Physics Research Laboratory, University of Michigan, Ann Arbor, MI 48109, U.S.A. \\ and \\ J. C. G. WALKER \\ Arecibo Observatory, National Astronomy and Ionosphere Center, Arecibo, PR 00612, U.S.A.
}

(Received in final form 7 July 1976)

\begin{abstract}
The visible airglow photometer on the Atmosphere Explorer C Satellite has been used to compare the calibrations of a number of ground-based airglow observatories. Discrepancies between different ground stations as large as a factor of six have been revealed. Efforts to account for these discrepancies have resulted in the discovery of differences as large as a factor of 2 in the standard light sources in use at different observatories. The participation of additional observatories in the intercomparison of standard sources is solicited. The project has also led to the discovery of a source of error that can amount to another factor of 2 in the procedure used to calibrate many airglow instruments. In the course of the project detailed maps, based on satellite cata, have been made of the galactic and zodiacal light background at a number of wavelengths, and a substantial source of contaminating emission has been discovered in the satellite data; the contamination appears to result from interaction of the spacecraft and the atmosphere at altitudes below $170 \mathrm{~km}$.
\end{abstract}

\section{INTRODUCTION}

The Atmosphere Explorer satellites carry a wide range of instruments designed to measure simultaneously both the neutral and ionic composition and temperatures of the atmosphere as well as airglow emissions, solar EUV fluxes, and fluxes of suprathermal charged particles. The satellites and their instrumentation have been described in a special issue of Radio Science published in April of 1973. The satellites are spin-stabilized, with the spin axis normal to the orbital plane, and a spinrate that can be controlled from the ground by means of a momentum wheel. The satellites carry on-board propulsion which makes it possible for them to maintain perigee altitudes as low as 130 $\mathrm{km}$ for extended periods of time.

During the planning of the program it was realized that the low perigee altitude of the satellites provided an excellent opportunity to intercalibrate ground-based airglow observatories around the world using the satellite airglow photometers. These instruments have been described by Hays ef al. (1973). They are fixed-filter instruments

* Also the National Institute for Telecommunications Research of the Council for Scientific and Industrial Research, Johannesburg, South Africa; and the National Astronomy and Ionosphere Center, Cornell University, Ithaca, NY, U.S.A. containing interference filters with nominal bandwidths of about $20 \AA$. The filters are mounted in a wheel that can be rotated under control from the ground to measure airglow surface brightnesses at the six different wavelengths listed in Table 1. There are eight positions of the filter wheel, the extra two positions providing for a measurement of the dark count and for calibration of the system, not including the filter, by means of a radioactive $\mathrm{Pm}_{147}$ source. Two optical channels, oriented at right-angles to the spin axis, permit simultaneous measurements at two different wavelengths. One channel has a narrow field of view designed to resolve airglow altitude profiles during horizon scans. The other channel has a wide field of view designed to provide high sensitivity for the measurement of airglow emissions in the zenith. Both channels are carefully baffled to prevent contamination of the measurements by sunlight or by light reflected from the sunlit Earth.

Airglow instruments on satellites prior to the Atmosphere Explorer program have not been useful for the intercalibration of ground-based observations because they have flown at altitudes above the airglow emission layers. Surface brightnesses measured from above are subject to a large and uncertain correction for the albedo of the ground. Atmosphere Explorer is the first satellite able to measure the surface brightness at $6300 \AA$ while 
TABle 1. AtMosPheric EMISSIONS OBSERVED BY AE-C

\begin{tabular}{cc}
\hline Wavelengths & Transition \\
\hline 3371 & $\mathrm{~N}_{2}\left(\mathrm{~B}^{3} \pi_{\mathrm{g}}-\mathrm{C}^{3} \pi_{u}\right)$ \\
4278 & $\mathrm{~N}_{2}^{+}\left(\mathrm{X}^{2} \Sigma_{\mathrm{g}}^{+}-\mathrm{B}^{2} \Sigma_{u}{ }^{+}\right)$ \\
5200 & $\mathrm{~N}\left({ }^{4} \mathrm{~S}^{2} \mathrm{D}\right)$ \\
5577 & $\mathrm{O}\left({ }^{1} \mathrm{D}^{1} \mathrm{~S}\right)$ \\
6300 & $\mathrm{O}\left({ }^{3} \mathrm{P}-{ }^{1} \mathrm{D}\right)$ \\
7319 & $\mathrm{O}^{+}\left({ }^{2} \mathrm{D}^{2} \mathrm{P}\right)$ \\
\hline
\end{tabular}

looking vertically upwards through the emission layer. It was this capability that we planned to exploit in order to intercalibrate ground-based airglow observatories in the most straightforward manner possible-comparison of the surface brightnesses at $6300 \AA$ measured by the satellite instrument with that measured by the ground-based instrument while both instruments are looking up at the same region of the sky.

This procedure does not, of course, provide any information on which airglow instruments correctly measure the surface brightness. Only a relative calibration between different instruments is established. It was felt, nevertheless, that even relative calibrations would contribute to the development of the global network of airglow observatories into a reliable source of synoptic information on thermospheric properties and behaviour.

Accordingly, we attempted to contact all of the airglow observatories around the world of which we had any knowledge. A list of the observatories that responded appears in Table 2; their geographic positions are shown in Fig. 1. This list is probably as accurate as any extant list of currently active airglow observatories. We would appreciate being notified of any errors or omissions.

This paper reports the results of the airglow intercalibration project. For reasons described below it turned out to be much more difficult to intercalibrate ground stations than had been anticipated. In this aspect, the project achieved very limited success. On the other hand, in the course of finding out why the intercalibration of groundstations is so difficult, we learned a good deal about possible sources of error both in ground-based and satellite airglow measurements. What we learned is presented in this paper in the hope that it may contribute to increased accuracy of future airglow observations.

\section{INTERCOMPARISON OF GROUND-BASED OBSER- VATORIES}

\subsection{Limited opportunities for coincident observations}

The intercalibration project called for coincident observations of the $6300 \AA$ airglow by satellite and ground-station with the satellite instrument looking up at the same region of the sky as the ground instrument. Such coincident observations proved remarkably difficult to achieve, for reasons that are presented in this section.

First, we were limited to times when the satellite was close to perigee, at altitudes below $200 \mathrm{~km}$, so that the satellite photometer could look up through the airglow layer. Second, ground-based airglow observations are possible only when the solar zenith angle exceeds about $100^{\circ}$. These two requirements together turned out to be very restrictive.

Figure 2 shows the development with time of the latitude and local time of the perigee of the Atmosphere Explorer C satellite (AE-C). Also shown

TABLE 2. Airglow STATIONS PARTICIPATING IN GROUNDBASED/AE-C PHOTOMETER INTERCOMPARISON

\begin{tabular}{|c|c|c|}
\hline \multirow[b]{2}{*}{ Station } & \multicolumn{2}{|c|}{ Geographic Coordinates } \\
\hline & Latitude & Longitude \\
\hline 1) Abastumani & 41.7 & 42.8 \\
\hline 2) Ann Arbor & 42.4 & -83.9 \\
\hline 3) Arecibo & 18.4 & -66.9 \\
\hline 4) Ashkhabad & 38.0 & 55.0 \\
\hline 5) Cachoeira Paulista & -22.7 & -45.2 \\
\hline 6) College & 64.9 & -148.0 \\
\hline 7) Fritz Park & 39.9 & -105.5 \\
\hline 8) Frunze & 43.0 & 74.0 \\
\hline 9) Gulmarg & 34.0 & 74.4 \\
\hline 10) Haute Provence & 43.9 & 5.7 \\
\hline 11) Kiruna & 67.8 & 20.4 \\
\hline 12) Kislovdsk & 44.0 & 43.0 \\
\hline 13) La Trobe & -37.4 & 144.1 \\
\hline 14) Leningrad & 59.9 & 30.3 \\
\hline 15) Liege & 50.6 & 0.4 \\
\hline 16) Lycksele & 64.6 & 18.8 \\
\hline 17) Loparskaya & 68.2 & 33.1 \\
\hline 18) Memambetsu & 43.9 & 144.3 \\
\hline 19) Montana & 48.6 & -107.1 \\
\hline 20) Mt. Abu & 24.6 & 72.7 \\
\hline 21) Niigata & 37.7 & 138.8 \\
\hline 22) Omakau & -45.0 & 169.6 \\
\hline 23) Poona & 18.0 & 73.0 \\
\hline 24) Richland & 46.3 & -119.4 \\
\hline 25) Sanae & -70.0 & -2.0 \\
\hline 26) Saskatoon & 52.1 & -114.1 \\
\hline 27) Sendai & 38.1 & 140.5 \\
\hline 28) Skibotn & 69.22 & 20.18 \\
\hline 29) Stara Jagora & 42.4 & 25.5 \\
\hline 30) Sutherland & -32.4 & 20.8 \\
\hline 31) Tzimliansk & 47.5 & 42.0 \\
\hline 32) Volgograd & 48.0 & 46.0 \\
\hline 33) Yakutsk & 62.0 & 129.7 \\
\hline 34) York & 43.8 & -79.9 \\
\hline 35) Zvenigorod & 55.7 & 36.8 \\
\hline
\end{tabular}






Fig. 1. Geographic DISTRIBUTION OF GROUND.BASED aIRgLOW OBSERVATORIES THat RESPONDED TO OUR REQUEST FOR PARTICIPATION IN THE INTERCALIBRATION PROJECT.

The stations are listed in Table 2.

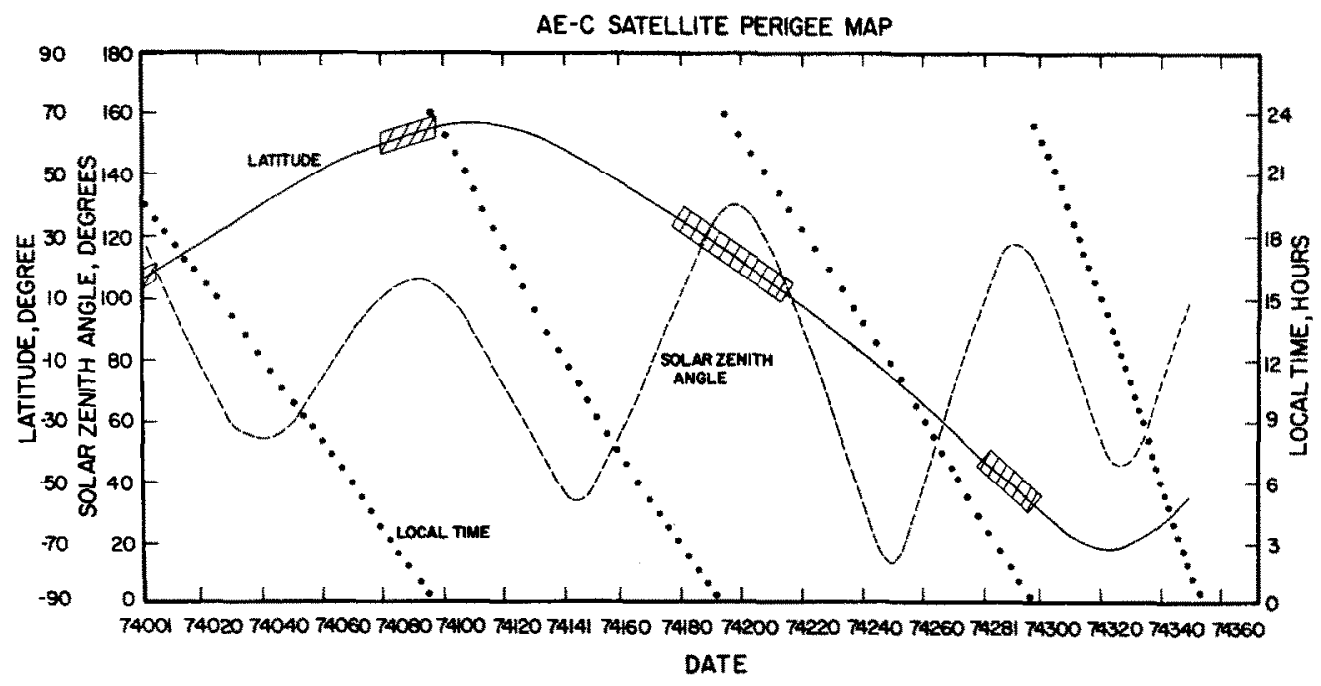

Fig. 2. Latitudinal VARIATION OF AE-C PERIGEe AS A FUNCTION OF DAY NUMBER IN 1974.

The cross-hatched areas show the only times when the solar zenith angle was large enough to permit coincident ground-based airglow observations. 
is the solar zenith angle at the perigee. The crosshatched boxes on the latitude curve show the only occasions when the satellite was below $200 \mathrm{~km}$ at night. At the end of $1974 \mathrm{AE}-\mathrm{C}$ entered a circular orbit at an altitude above $200 \mathrm{~km}$, precluding further attempts at coincident airglow observations.

Figure 2 shows that the nature of the AE-C orbit was such as to permit coincident airglow observations only over restricted latitude ranges. The many observatories located outside these latitude ranges could not be included in the intercalibration project. Furthermore, coincident observations were possible only during relatively short periods of time, as shown in Fig. 2, and during these short periods it was necessary for conditions of weather and moonlight to be favorable for ground-based airglow measurements, for the satellite to pass very nearly over the ground stations, and for both satellite and ground station to make the right measurements.

In spite of our best efforts, the number of occasions on which all of these conditions were satisfied was not large. Unfortunately there was another phenomenon, completely unexpected, that rendered useless many of the coincident observations that were achieved. This phenomenon is described in the next section.

\subsection{Contamination of satellite airglow meas- urements at low altitude}

Coincident airglow observations were carefully scheduled with the satellite at the lowest possible altitudes so as to insure that the satellite would be below the $6300 \AA$ emission layer. It was not realized, until well into the mission, that the airglow measurements are contaminated by an unexplained interaction with the neutral atmosphere at altitudes below about $170 \mathrm{~km}$. The contamination appears as an increase in the measured surface brightness that is approximately proportional to the ambient atmospheric density. The increase is greatest when the instrument is looking into the direction of motion of the satellite, as shown in Fig. 3, but the effect is present at heights below $170 \mathrm{~km}$ even when the instrument is looking vertically upwards.

Figure 3 is an illustrative contour plot of the measured surface brightness at $7319 \AA$ as a function of altitude and the zenith angle of the photometer, which is rotating in the orbital plane. The contamination is present at all the wavelengths observed on AE-C; it appears to increase in intensity towards longer wavelengths.

We do not yet understand this phenomenon and

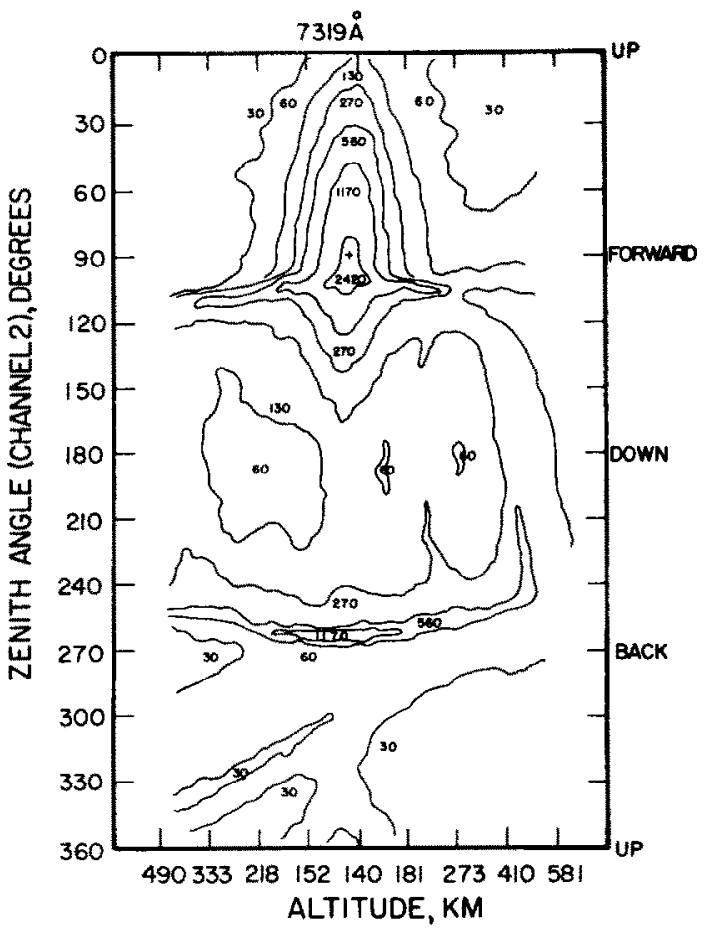

Fig. 3. Illustration OF THE ENHANCEMENT OF MEASURED SURFACE BRIGHTNESS AT PERIGEE.

Plotted are contours of measured surface brightness in Rayleighs at $7319 \AA$ as a function of photometer zenith angle and altitude.

can only speculate that NO, entering the instrument at a relative velocity of about $8 \mathrm{~km} \mathrm{sec}$, recombines with $\mathrm{O}$ on the walls of the instrument to produce emission in the $\mathrm{NO}_{2}$ continuum. The effect of the contamination on the airglow intercalibration project has been severe, however, because it has made it necessary to discard otherwise successful coincident observations made with the satellite below $170 \mathrm{~km}$.

\subsection{Results of the intercomparison}

Because of the hazards and difficulties described above the number of successful coincident observations was reduced to nine. In Fig. 4 we show the ratio of the surface brightness at $6300 \AA$ measured on the ground to that measured on the satellite as a function of date. The values plotted represent the surface brightnesses reported to us by the groundbased observers without corrections introduced by subsequent comparison of standard sources or second thoughts about extinction. They are presumably representative of the accuracy that can be expected of measurements of airglow surface brightness in the absence of special efforts to insure consistency. 


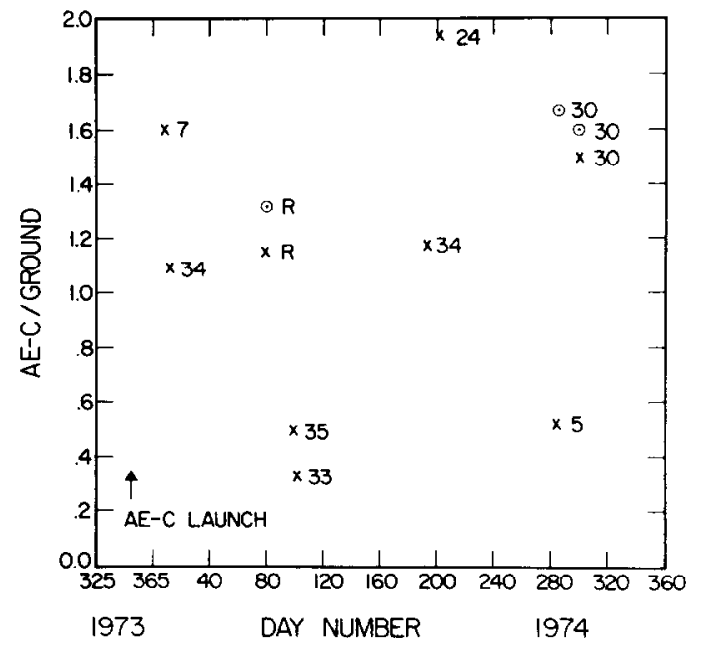

Fig. 4. ThE RATIO OF THE SURFace BRightNess AT $6300 \AA$ MEASURED BY AE-C TO COINCIDENT GROUND-BASED MEASUREMENTS AS A FUNCTION OF DAY NUMBER IN 1974.

The ground-based observatories are identified in Table 2. Also shown, $\odot$, are some comparisons at 5577 discussed in Section 4.4 .

As already noted, we cannot say which measurements are correct and which ones are not, but the results in Fig. 4 show differences between different ground-based observatories, relative to the $\mathrm{AE}$ photometer, amounting to a factor of 6 . We must conclude either that the sensitivity of the satellite instrument varied markedly with time or else that some ground-based airglow measurements are seriously wrong. In the next section we describe our efforts to ensure precision in the satellite airglow measurements.

\section{RELIABILITY OF SATELLITE MEASUREMENTS}

\subsection{Galactic and zodiacal light}

Measurements with a fixed-filter airglow photometer like the AE instrument are subject to contamination by any sources of light that lie within the pass-band of the filter. Sources of contamination for ground-based instruments include $\mathrm{OH}$ and continuum emissions arising at mesopause heights as well as galactic and zodiacal light. Low-altitude airglow contamination is not a source of error in the satellite data, obtained above the mesopause, but careful correction for contamination by extraterrestrial light sources is necessary.

In order to make this correction we have used the satellite photometers at high altitudes to measure the brightness of the extraterrestrial emission free of airglow contamination. These measurements have made it possible to prepare maps of galactic surface brightness as a function of declination and right ascension and the surface brightness of the zodiacal light as a function of ecliptic latitude and longitude. One of these maps is shown, in preliminary form, in Fig. 5. With maps like this one, made with the AE instruments at the various wavelengths of interest, it is possible to correct the satellite airglow data for extraterrestrial emissions without introducing significant error. In the remainder of this section we describe the procedures used to obtain maps of extraterrestrial emission free of contamination.

Data to be used in the maps of extraterrestrial surface brightness must be obtained at altitudes above significant atmospheric emission but below the level where energetic particles in the radiation belts might affect the instrument.

Generally, the $6300 \AA$ airglow emission at night cannot be detected above the galactic background at heights exceeding $450 \mathrm{~km}$. During the day, the emission can be seen as high as $500 \mathrm{~km}$. We have therefore taken these two altitudes as the lower limits for observations of extraterrestrial emission. In addition, we have used only data taken with the photometer looking into the upward hemisphere. In the South Atlantic region, the radiation belt particles are encountered at very low altitudes (see for example, Fig. 1 of Torr et al., 1975). Figure 6, from Fowler et al. (1968), is a global map of cathode dark current from the OGO satellite photometers. The data used to produce this map were obtained from a photomultiplicr tube with a sapphire end window. The effect of the lowering of the radiation belts in the South Atlantic is clearly seen in the case of this PMT as a dramatic increase in the dark current. Using this map as a guide, we have rejected data obtained between geographic longitudes $100^{\circ} \mathrm{W}$ and $50^{\circ} \mathrm{E}$ and between geographic latitudes $15^{\circ} \mathrm{N}$ and $55^{\circ} \mathrm{S}$. We have set 1000 $\mathrm{km}$ as the upper altitude limit outside this region. There are indications, however, that these limits are overcautious in the case of AE-C. The AE photomultiplier appcars to be well shielded from the radiation environment, and preliminary maps of dark-count, similar to Fig. 6, show no pronounced effect of the radiation anomaly. In addition, the fused silica windows of the AE photomultipliers are expected to yield much less fluorescence than the sapphire end window of the OGO photomultiplier.

The data on extraterrestrial emission must be free not only of contamination by airglow and energetic particles but also by sunlight, moonlight, and light from the bright Earth scattered off the instrument itself. $\mathrm{A}$ feature of the $\mathrm{AE}$ satellite 


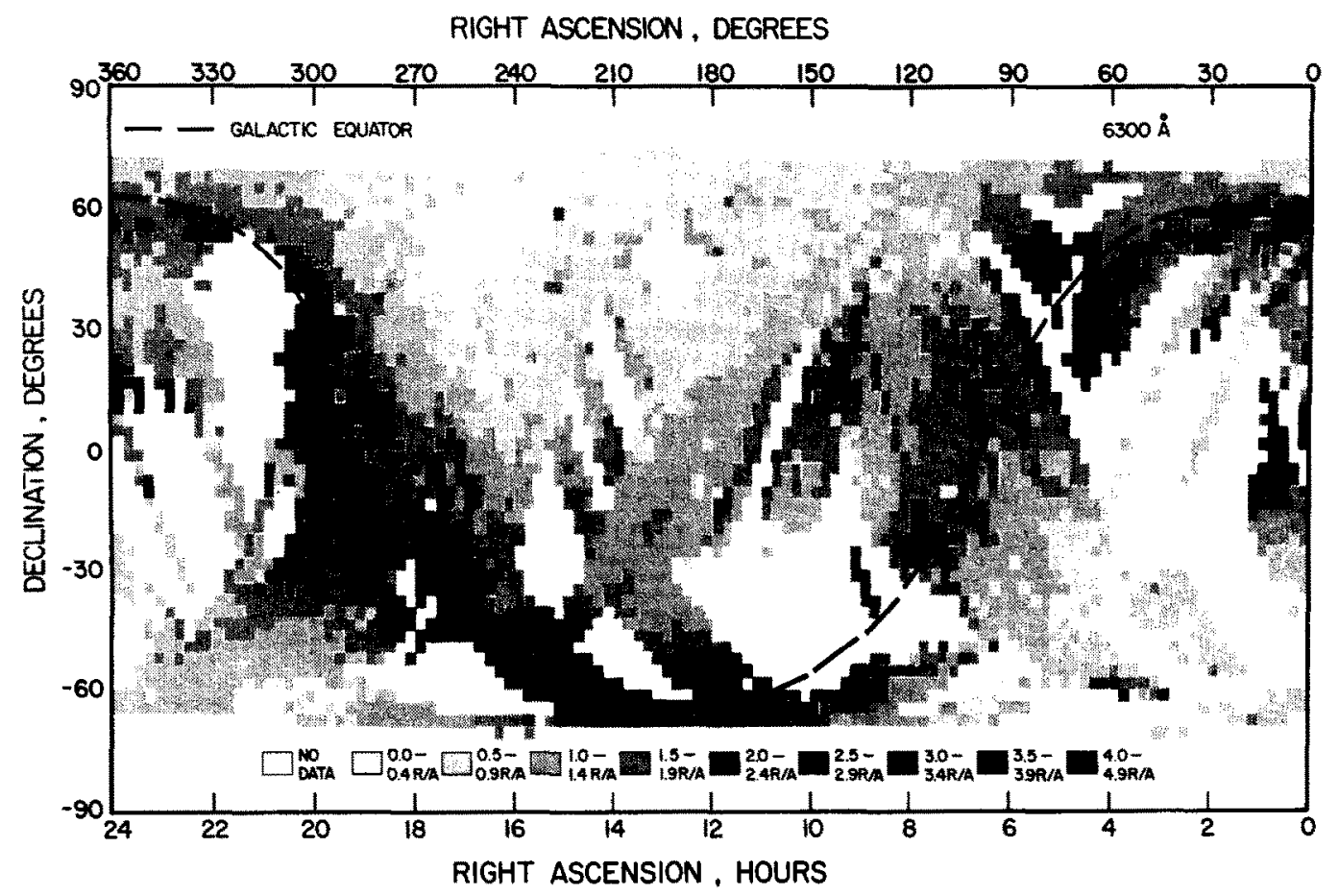

Fig. 5. MAP of GALACTiC surface bRightness IN R/A MEASUREd by the AE-C PHotometer at $6300 \AA$.

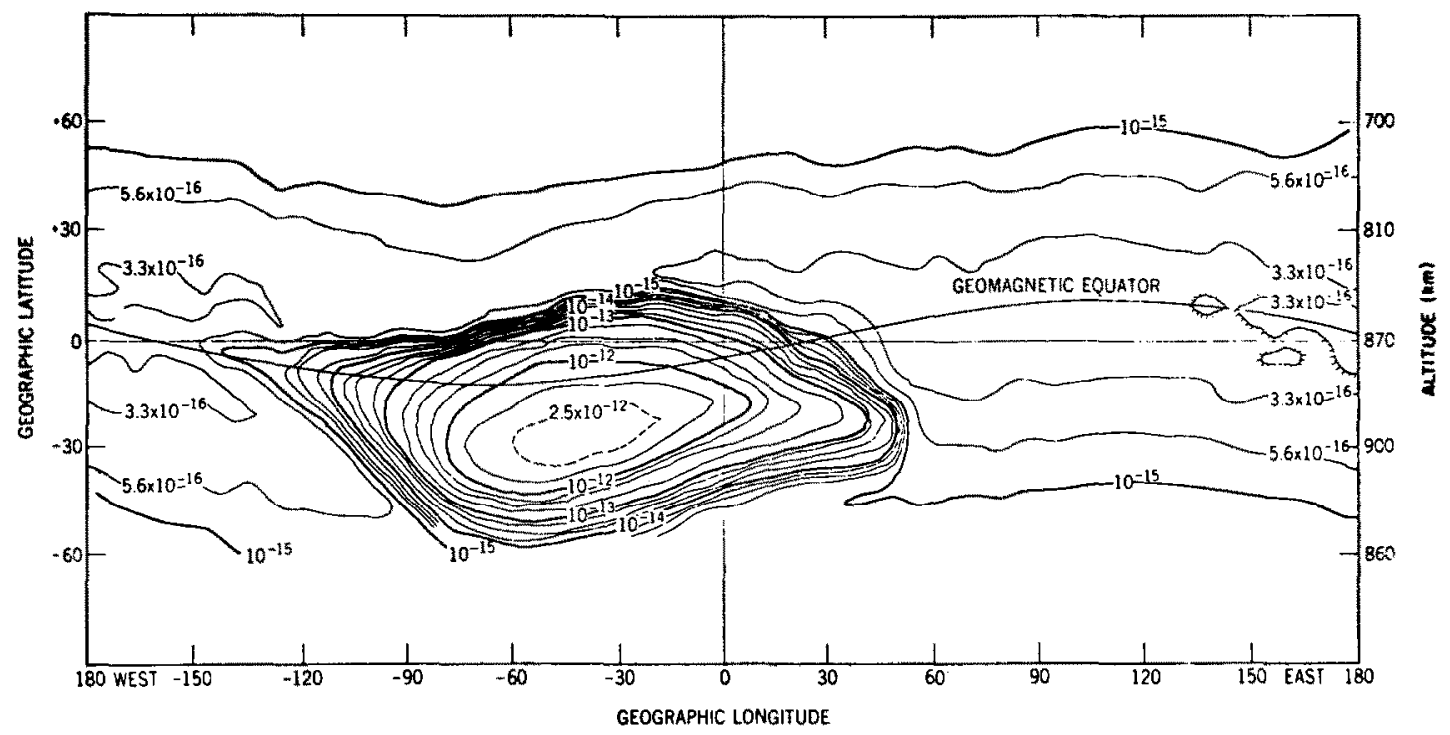

Fig. 6. Global MAP OF CATHODE DARK CURRENT IN OGO PHOTOMETERS SHOWING EFFECTS OF THE RADIATION BEI T AND THE SOUTH ATt.ANTIC ANOMALY (FROM Fowler et al., 1968). 


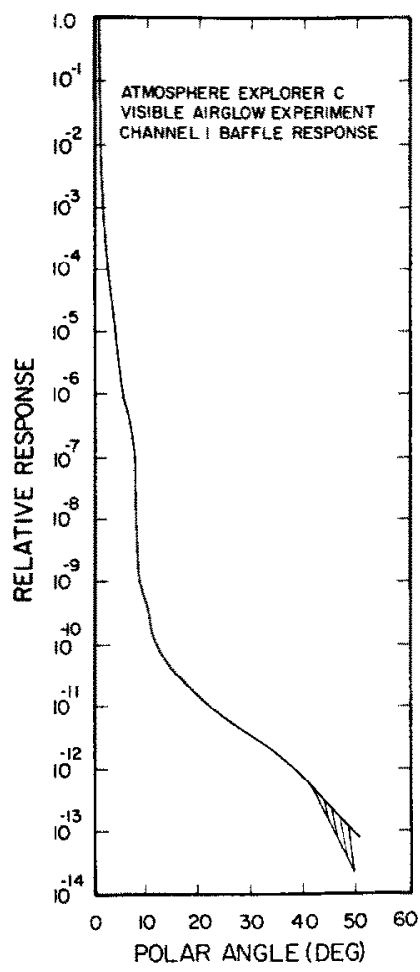

FIG. 7. LABORATORY MEASUREMENTS OF THE RESPONSE OF THE AE-C PHOTOMETER, WITH BAFFL, TO OFF-AXIS RADIATION.

The polar angle is the angle between the line of sight of the instrument and the light source.

photometer is the two stage baffle system described by Hays et al. (1973). This is designed to attenuate scattered light from outside the field of view by a factor of $10^{-12}$ (Fig. 7), making it possible to measure the galactic background (at a typical surface brightness of $30 \mathrm{R}$ ) even in the presence of sunlight (equivalent to $10^{13} \mathrm{R}$ if viewed directly). In preparing the galaxy map we have not used data taken within $45^{\circ}$ of the Sun and $20^{\circ}$ of the Moon, or data obtained at a photometer zenith angle larger than $90^{\circ}$.

The surface brightness map at $6300 \AA$ is shown in Fig. 5. Data have been averaged in $3^{\circ}$ by $3^{\circ}$ bins. The units are Rayleighs per angstrom, as we have divided by the effective bandwidth of the filter to convert from Rayleighs. At the time of launch the $6300 \AA$ filter bandwidth was $23 \AA$, and we have to assume that the bandwidth remained constant over the period during which the data comprising this map were taken. The assumption is reasonable since the most likely change in the filter characteristics, if any, is a shift in wavelength of peak transmission rather than change in bandwidth or peak transmission. A wavelength shift does not degrade the galaxy results as the galaxy is effectively a broadband source.

No attempt has been made to interpolate across regions for which we do not yet have data, as these will be filled as more of the AE-C data base is processed. The AE-C satellite had an inclination of $68.4^{\circ}$ and so never reached the higher declinations. There is some contamination due to the zodiacal light at low ecliptic latitudes in Fig. 5. This will be extracted in the final maps, which we expect to publish as part of a study of galactic and zodiacal light surface brightnesses at six different wavelengths.

\subsection{Stability of photometer gain}

The major cause of degradation in the gain of photomultipliers is exposure to high dynode current. A sophisticated protection system has been incorporated into the AE photometers to alleviate this problem. In addition, a calibration source of phosphor activated by radioactive promethium ${ }_{147}$ is mounted in the filter wheel and used to check the electronics on each orbit. A monitor of the photometer response to this source over the lifetime of the instrument can provide a check on the detector stability, provided that we know the decay of the radioactive source with reasonable accuracy.

Measurements of the intensity of this source using the AE-C photometer were made over a period of 10 months prior to launch. These measurements are shown in Fig. 8. The measured intensity of the calibration source over a period of 18

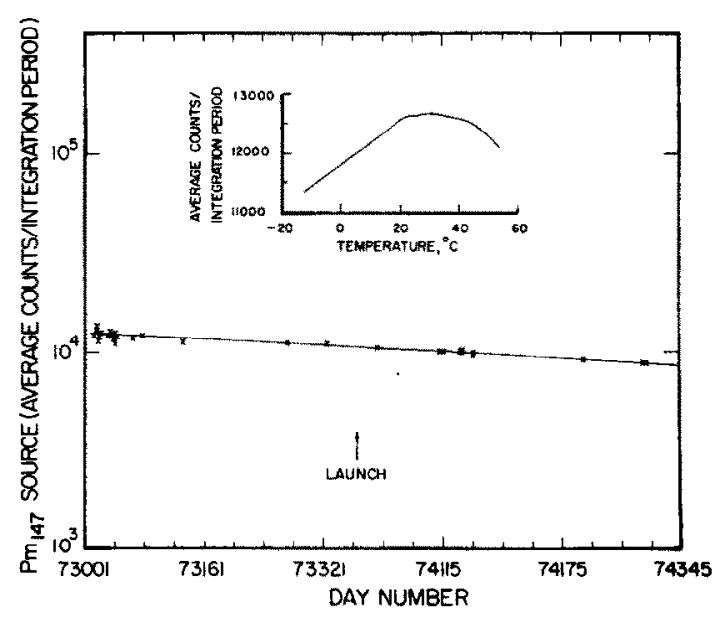

Fig. 8. Brightness of The $\operatorname{Pm}_{147}$ source as a function OF TIME.

The inset shows laboratory measurements of the variation with temperature in the brightness of the source. 
months after launch has been added as an extension to the prelaunch measurements. From the figure it can be seen that the source continued to decay with the prelaunch half-life of $4.9 \mathrm{yr}$.

An additional check on the photomultiplier involves cycling the discriminator level from normal to a higher voltage while the $\mathrm{Pm}_{147}$ source is in position. The difference in signal has remained at the prelaunch level. From these two checks it can be concluded that the electronics, including the photomultiplier, have remained stable.

Of interest, however, in the use of radioactive phosphor sources is the fact that the half-life of the $\mathrm{Pm}_{147}$ source is almost twice the half-life of pure $\mathrm{Pm}_{147}$, which is $2.5 \mathrm{yr}$. The finding that the brightness of a radioactive phosphor does not decay with the same half-life as the radioactive material embedded in the phosphor is supported by earlier studies by Smith and Alexander (1963) and Blacker and Gadsen (1966). The variation in the intensity of the source with temperature was measured in the laboratory prior to launch; this variation (shown in Fig. 8) was found to be not more than $8 \%$ over the temperature range $-10^{\circ} \mathrm{C}$ to $+50^{\circ} \mathrm{C}$.

\subsection{Stability of satellite interference filters}

The results described in the preceding section demonstrate the stability of the AE-C photometers, excluding the filters. If the sensitivity of the satellite instrument changed with time it must have been due to changes in the interference filters.

Every effort was made in the design and construction of the filters to ensure that they would remain stable. No colored glass blocking elements which could be darkened by radiation were used in their manufacture. Also no epoxy bonding was used between the substrate layers. Therefore, unless the filters were contaminated by moisture leaking into them before launch (the moisture subsequently being lost in flight with accompanying change in transmission characteristics) the filters should, in principle, have remained stable. However, the satellite photometer does not have an inflight standard source that calibrates through the filters, so we have no direct check of the filter transmission characteristics over the inflight lifetime of the instrument.

Reed et al. (1971) used bright stars to check the stability of the interference filters of the OGO-6 satellite photometers. Their results show a decrease in transmission over a period of 11 months in orbit by a factor of 1.65 . The OGO filters, however, were not constructed to minimize radiation darkening, so these results do not imply that the AE filters should have degraded to the same extent. As stars are basically white light sources, the procedure used on OGO gives information only on the total transmission of the filter and not on shifts in the wavelength of peak transmission.

In order to check the long-term stability of the AE filters, therefore, we decided to turn the ground-station intercalibration project around and use results of coincident measurements with a few ground-based instruments that had been carefully intercalibrated in the laboratory. In the course of this investigation, described in the next section, we discovered several possible sources of error in ground-based airglow measurements that may have contributed to the marked disagreement between individual observations that appears in Fig. 4.

\section{LONG TERM STABILITY OF AE FILTERS}

\subsection{Comparison of standard light sources}

The first step was to compare, in the laboratory, the standard sources used at a number of groundbased observatories and the AE source.

The standard light source used in the preflight absolute calibration of the AE photometers is a tungsten strip filament lamp calibrated by the $\mathrm{Na}$ tional Bureau of Standards, Boulder, Colorado. We compared this source in the laboratory against 10 other standard sources from various airglow observatories. The comparisons were made using the photometers built for flight on AE-D and AE-E.

The ratio of the brightness of each source as determined from the $A E$ source to the brightness determined at the previous calibration of each source is shown in Fig. 9. As in the case of the ground-station intercomparison, we do not know which calibration is correct. The AE source is being used, here, only as a basis for comparison. While many of the sources agree to within $30 \%$, differences at some wavelengths are as much as a factor of two. The original calibrations of several of the sources for which results are shown in Fig. 9 can be traced, like the AE source, to the National Bureau of Standards. If we assume that the NBS calibration has remained constant we may conclude that the brightness of at least some sources has varied with time. The results show the need for periodic intercomparisons of sources if comparisons of airglow intensities between one observatory and another are to be meaningful.

\subsection{Substantial source of error in calibration of some} airglow photometers using extended light sources

When intercalibrating the standard light sources 


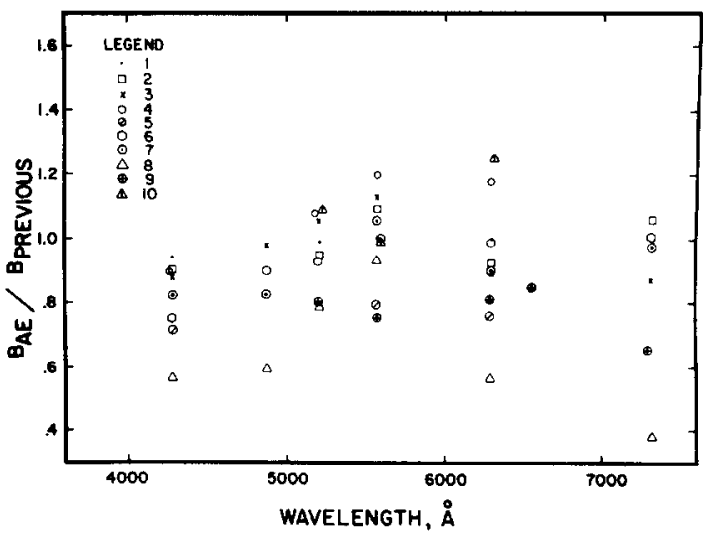

Fig. 9. Ratio of THE BRIGHTNESSES OF VARIOUS CALIBRATION SOURCES DEDUCED FROM THE AE STANDARD SOURCE TO BRIGHTNESSES DERIVED FROM EARLIER CALIBRATIONS OF THESE SOURCES.

The sources are

(1) Ann Arbor, quartz iodide (QI)

(2) Sutherland, $\mathrm{C}_{14}$

(3) Arecibo, $\mathrm{C}_{14}$

(4) Arecibo, QI

(5) Calgary (ISIS source) QI

(6) Fritz Peak, $\mathrm{C}_{14}$

(7) Ann Arbor, $\mathrm{C}_{14}$

(8) York, $\mathrm{C}_{14}$

(9) Uppsala, $\mathrm{C}_{14}$ (a)

(10) Uppsala, $\mathrm{C}_{14}$ (b)

in the laboratory, we initially used an optical configuration which pointed out an error made in the calibration of many airglow photometers. A check of filter sideband leakage was made by placing in the optical path a second interference filter of the same wavelength as that in the photometer. The second filter was placed over the entrance to the photometer, with the phosphor source immediately following it (see Torr et al., 1976a). This configuration had the effect of making the photometer appear to be more sensitive than it was with a single filter.

The reason for this spurious result is that the interference filter acts in combination with the white phosphor as a resonant cavity. Light rejected by the filter, which has a semimirrored surface, is reflected between the surface and the white phosphor, effectively increasing the source brightness. Figure 10 is from another paper (Torr et al., 1976a) in which we discuss this effect. The figure compares laboratory measurements of the enhancement with theoretical calculations for a range of typical filter transmissions.

It can be seen from these results that serious calibration errors (a factor of $\sim 2$ ) can arise when a calibration source with a reflecting surface is placed close to an interference filter. Most of the airglow measurements made during the IGY and for years afterwards used the Roach turret photometer (Purdy et al., 1961), in which the filters rotate directly under and close to a built-in phosphor source. As a result, multiple reflections between filter and source make the instrument appear more sensitive than it really is; the airglow intensity is underestimated. This error, of course, also affects the measurements made with other types of photometers calibrated against these instruments.

In Fig. 11 we compare $6300 \AA$ airglow surface brightness measured by a turret photometer at Arecibo Observatory with simultaneous measurements by a tilting filter photometer not subject to this calibration error. The results clearly illustrate the effect. In the turret photometer used here the separation between filter and source was $1.1 \mathrm{in}$. and the radius of the filter was 1.5 in., yielding a value of 0.73 for $L / r$.

From the slope of the line in Fig. 11, equal to $B_{\text {source }} / B_{\text {enhanced }}$, we obtain the circled point plotted in Fig. 10. Agreement of the field measurements with laboratory measurements and theory is good. There is no reason to doubt that this source of error affects the calibration of many airglow observatories around the world. A filament source with a diffuser plate made, for example, from fused silica



FIG. 10. ILLUSTRATION OF ERROR INTRODUCED WHEN THE CALIBRATION SOURCE IS TOO CLOSE TO THE INTERFERENCE FILTER.

The crosses are laboratory measurements while the curves are the theoretical (see Torr et al., 1976a) ratio of the apparent to the real brightness of the source as a function of $L / r$, where $r$ is the filter radius and $L$ is the distance between the filter and the source. The reflectance of the phosphor is $R$, and the transmission of the filter is $T$. The circled dot shows the result of a comparison, in the field, of airglow measurements by a turret photometer with $L / r<1$ and a tilting filter photometer with large $L / r$. 




FIG. 11. COMPARISON OF AIRGLOW SURFACE BRIGHTNESS IN R MEASURED BY A TURRET PHOTOMETER (CALIBRATION SOURCE CLOSE TO FILTER) AND A TILTING FILTER PHOTOMETER (OPAL SCREEN OF SOURCE 7 in. FROM FILTER.

will give rise to the same error. The results shown in Fig. 2 indicate that the source should be a distance of at least five filter radii from the interference filter.

Another source of calibration error has recently been pointed out by Muscari (1975). When the source is placed inside the hyperfocal distance there is an increase in the effective field of view of the instrument through vignetting resulting in an overestimation of the source brightness of up to $20 \%$.

\subsection{Stability at $6300 \AA$}

In view of the possibility of large discrepancies between the standard light sources of different observatories, we have used, for establishing the longterm stability of the satellite filters, only observatories whose sources have been compared in the laboratory with the AE source. Laboratory comparison allows us to normalize the calibrations. We have also used only data not subject to the calibration error discussed in Section 4.2. Four successful comparisons with intercalibrated ground stations were achieved. In Fig. 12 we show the ratio of the $6300 \AA$ surface brightness measured on the satellite to that measured on the ground. The satellite data entering these ratios have been corrected for galactic background, sensitivities have been normalized by the ratio of standard sources shown in Fig. 9, and the ground observations have been corrected for extinction in the lower atmosphere.

In correcting for atmospheric extinction we have used the attenuation coefficients tabulated by Elterman (1968) and McClatchey et al. (1972) for clear air. If $I_{0}$ is the intensity above the troposphere and ozone layer and $I_{\text {obs }}$ is the intensity observed in the zenith from the ground

$$
I_{0}=I_{\text {obs }} \mathrm{e}^{\tau_{a}}\left[\mathrm{e}^{-\tau}+\mathrm{g}\left(1-\mathrm{e}^{-\tau}\right)\right]^{-1},
$$

where $m$ is the mass of air through which the transmitted light passes and is unity in the zenith at sea level;

$\tau$ is the total optical thickness due to molecular $\tau_{m}$ and aerosol $\tau_{a}$ scattering and to absorption

$$
\begin{aligned}
& {\left[\tau=\left(\sigma_{m}+\sigma_{\alpha}+k_{0}+k_{\alpha}\right] m\right.} \\
& =(0.06+0.232+0.035+0.007) m=0.317
\end{aligned}
$$

where $\sigma_{m}$ and $\sigma_{a}$ are the scattering thicknesses for molecules and aerosols and $k_{0}$ and $k_{a}$ are the absorption thicknesses for ozone and aerosols);

$\tau_{a}=\left(k_{0}+k_{a}\right) m=0.042$.

$g$ is a fraction determined by the directional distribution of the scattered radiation and by the amount of multiple scattering. We have used $g=$ 0.5 here. However, increased forward scattering tends to increase $g$ while multiple scattering, diffusion, or absorption by particulate matter decreases $g$ (Leighton, 1961).

Thus, for a clear atmosphere the extinction correction at $6300 \AA$ can amount to $20 \%$, but significantly larger corrections may be appropriate for increased particulate matter or thin cirrus in the sky. The ratios shown in Fig. 12 are therefore upper limits, as we have used the clear atmosphere aerosol model here, and the aerosols vary markedly with time both in concentration and nature.

Also shown in Fig. 12 is a comparison made in an aurora between the AE-C satellite and a photometer flown on a rocket from Fort Churchill on 21 March 1974. In this case no extinction correction was necessary, and the rocket photometer had been



Fig. 12. RATIO Of THE AIRGLOW SURFACE bRIGHTNESS AT $6300 \AA$ MEASURED BY THE AE-C PHOTOMETER TO COINCIDENT SURFACE BRIGHTNESS MEASURED BY GROUND-BASED INSTRUMENTS AND A ROCKET-BORNE PHOTOMETER.

The satellite measurements have been corrected for extraterrestrial contamination, the ground-based measurements have been corrected for extinction, and the ratio has been corrected for differences in calibration sources. Sources of the coincident data are: (1) Fritz Peak; (2) York; (3) Fort Churchill rocket; (4) Sutherland. 
calibrated against the AE source. The agreement is within $20 \%$.

The results show that the sensitivity of the AE-C satellite photometer, including the filter, has remained stable at $6300 \AA$.

\subsection{Stability at $5577 \AA$}

With the AE-C perigee never going below 130 $\mathrm{km}$, the satellite was always above the lower altitude $5577 \AA$ emission. As a result it was not possible to compare directly the $5577 \AA$ surface brightness measured by $\mathrm{AE}-\mathrm{C}$ and by a ground photometer with both instruments looking vertically up through the emission. In order to make a comparison at this wavelength, use was made of the variable spin rate of the spacecraft. Two orbits were selected which passed over the tilting filter photometer at Sutherland $\left(32.4^{\circ} \mathrm{S}, 20.4^{\circ} \mathrm{E}\right)$ near perigee. On these orbits, the satellite was spun at $2 \% \mathrm{sec}, \frac{1}{12}$ of its normal spin rate. At this spin rate the narrow channel ( $\frac{30}{4}$ half angle field of view) was able to scan through the low altitude $5577 \AA$ emission with sufficient resolution for the slant profile to be obtained. In Fig. 13 we show an example of intensity measured as a function of photometer zenith angle. For each orbit two horizon scans were used, one just south of the ground facility with the satellite photometer looking north across the sta-

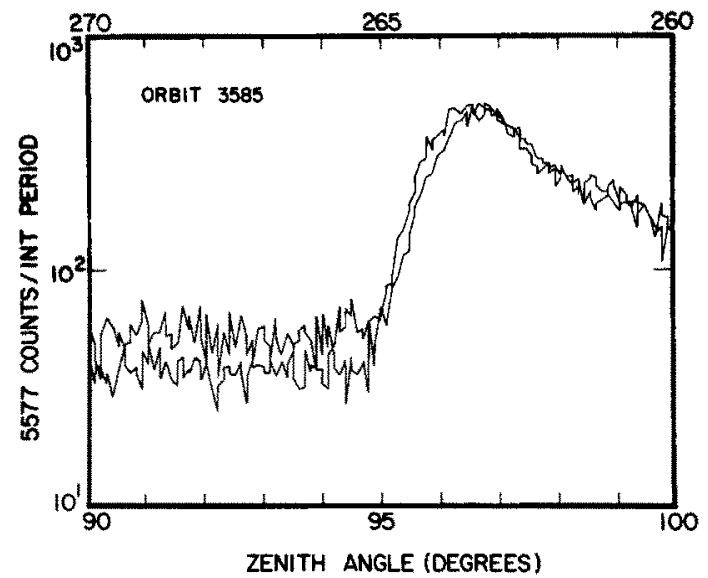

Fig. 13. EXAMPLES OF HORIZON SCANS OF THE LOW ALTITUDE GREEN LINE EMISSION MEASURED OVER SUTHERLAND BY THE NARROW ANGLE PHOTOMETER ON AE WITH THE SATELLITE SPINNING SLOWLY.

One scan looks forward as the satellite approaches the ground-station; the other looks back as the satellite recedes. After correction for dark count and extraterrestrial background the two scans are averaged and inverted to obtain the height profile of the volume emission rate over the ground station. tion, and one to the north looking back across the ground station. These two spins were averaged and inverted to yield vertical intensities. The results are summarized in Table 3 . When the satellite intensities are corrected for the $6 \%$ difference in the standard light sources at $5577 \AA$ and the ground observations corrected for extinction, the agreement is better than $25 \%$. Galactic contamination is negligible for $5577 \AA$ airglow viewed tangentially.

We were also able to compare the AE-C satellite with the rocket photometer at $5577 \AA$ on the same occasion, early in the mission, for which the $6300 \AA$ comparison was made. In this case, the ratio $15577_{\mathrm{AE}-\mathrm{C}} / \mathrm{I} 5577_{\text {rocket }}$ was 1.32. Again the results indicate that the sensitivity of the AE photometer, including the $5577 \AA$ filter, varied by less than 20 per cent over a period of almost a year in orbit.

\section{CONCLUSIONS}

Coordinated measurements of airglow surface brightnesses at $6300 \AA$ and $5577 \AA$ by the $\mathrm{AE}$ photometers, a rocket photometer and intercalibrated groundbased observatories, combined with other tests described in this paper, have established that the satellite instrument has not varied significantly with time. Therefore, the very wide discrepancies revealed in Fig. 4 between surface brightnesses measured at different ground-based observatories and coincident satellite measurements can not be attributed to variability of the $\mathrm{AE}$ photometers.

As noted several times already, the procedures we have followed do not permit a determination of which measurements are correct. Evidently, however, measurements by some ground-based observatories differ from measurements by others by as much as a factor of six. More attention must be devoted to intercalibration before airglow data obtained at different ground-based observatories can be usefully combined to obtain synoptic information on the global state of the upper atmosphere.

In the course of carrying out our program of intercalibration of ground observatories with the Atmosphere Explorer satellite, we have found that the use of the satellite instrument as a transfer standard is very difficult. From the point of view of the absolute calibration of the ground instruments, the results show that agreement between different observatories can be achieved provided standard sources are intercompared, provided these sources are used to calibrate the photometers in a way that is free of known sources of error, and provided the photometers are capable of discriminating against 


\begin{tabular}{|c|c|c|c|c|c|c|}
\hline Orbit & Day No. & $\begin{array}{c}\text { I5577 } \\
\text { Sutherland } \\
\text { (R) }\end{array}$ & $\begin{array}{l}15577 \\
\text { AE-C } \\
\text { (R) }\end{array}$ & $\begin{array}{c}\text { I5577 } \\
\text { AE-C } \\
\text { (source } \\
\text { normalization) } \\
(\mathrm{R})\end{array}$ & $\begin{array}{c}15577 \\
\text { Sutherland } \\
\text { (corrected } \\
\text { for } \\
\text { extinction) } \\
\text { (R) }\end{array}$ & $\frac{15577_{\text {AE-C }}}{15577_{\text {Sutherland }}}$ \\
\hline $\begin{array}{l}3585 \\
3642\end{array}$ & $\begin{array}{l}74286 \\
74290\end{array}$ & $\begin{array}{r}135 \\
70\end{array}$ & $\begin{array}{l}226 \\
112\end{array}$ & $\begin{array}{l}212 \\
105\end{array}$ & $\begin{array}{r}174 \\
90\end{array}$ & $\begin{array}{l}1.21 \\
1.17\end{array}$ \\
\hline
\end{tabular}

cortaminating atmospheric and extraterrestrial emission. Coordinated measurements such as those described here do, however, have useful potential for further investigations concerning absorption and extinction in the lower atmosphere. Such measurements also can provide valuable information concerning the calibration of satellite photometers not available by other means. High quality, well calibrated ground airglow facilities in conjunction with satellite projects, can play a valuable scientific role, as the two modes of operation are complementary.

Accordingly, we propose to continue the intercalibration project; for purposes of improving the ground-based data we will undertake to provide a calibration, relative to an NBS calibrated lamp at The University of Michigan, of any standard source sent to us. This procedure will at least yield mutually consistent results. However, it is also necessary that regular checks be made of the transfer functions of the filters in the instruments.

Acknowledgements-Many people contributed to the results presented in this paper. Firstly, we are grateful to the many scientists at ground-based airglow observatories who were eager to participate in the intercalibration effort. We are also grateful to L. L. Cogger for the data shown in Fig. 11 and to W. Sharp for the rocket observations at the time of the coincident $\mathrm{AE}-\mathrm{C}$ overfight. We thank G. R. Carignan, D. G. Torr, and J, Meriwether for helpful discussions. This work was supported in part by NASA Contracts NAS 5-20705 and NSG 7104 to Cornell University. The National Astronomy and lonosphere Center is operated by Cornell University under contract with the National Science Foundation. One of the authors (J. C. G. Walker) thanks the Ionosphere Research Laboratory, The Pennsylvania State University, for providing an opportunity to work on this paper.

\section{REFERENCES}

Blacker, V. H. and Gadsden, M. (1966). On the calibration of airglow photometers, Planet. Space Sci. 14, 921-928.

Elterman, L. (1968). UV, Visible and IR attenuation for altitudes to $50 \mathrm{~km}$. ARCRL-68-D153, April.

Fowler, A. B., Reed, E. I. and Blamont, J. E. (1968). Effect of energetic particles on photomultipliers in earth orbits up to $1500 \mathrm{~km}$. Goddard Space Flight Center Report X-613-68-486, December.

Hays, P. B., Carignan, G. R., Kennedy, B. C., Shepherd, G. G. and Walker, J. C. G. (1973). The visible-airglow experiment on Atmosphere Explorer. Radio Sci. 8, 369-378.

Leighton, P. A. (1961). Photochemistry of Air Pollution. Academic Press, New York.

McClatchey, R. A., Fenn, R. W., Selby, J. E. A., Volz, F. E. and Garing. J. S. (1972). Optical properties of the atmosphere. AFCRL-72-0497, Environmental Research Papers, No. 411.

Muscari, J. A. (1975). Photometer calibration problem for extended astronomical sources. Appl. Optics 14, 14911492.

Purdy, C. M., Megill, L. R. and Roach, F. E. (1961). A new airglow photometer. J. Res. NBS (C)65, 214.

Reed, E. I., Fowler, W. B. and Blamont, J. E. (1972). An atlas of low latitude $6300 \AA$ (OI) night airglow from OGO-4 observations. Goddard Space Flight Center Report X-625-72-171, May.

Smith, L. L. and Alexander, R. B. (1963). IQSY Instruction Manual, No. 5, Part II, p. 20. IQSY Secretariat, London.

Torr, D. G., Torr, M. R., Walker, J. C. G. and Hoffman, R. A. (1975). Particle precipitation in the South Atlantic geomagnctic anomaly. Planet. Space Sci. 23, 15-26.

Torr, M. R., Hays, P. B., Kennedy, B. C. and Tor, D. G. (1976a). Discussion of a sigxificant photometer calibration error using extended standard sources. Appl. Optics 15, 600-602.

Torr, M. R., Burnside, R. G., Hays, P. B., Stewart, A. I., Torr, D. G. and Walker, J. C. G. (1976b). Metastable ${ }^{2} \mathrm{D}$ atomic nitrogen in the midlatitude nocturnal ionosphere. J. geophys. Res. 81, 531-537. 